\title{
A Spreadsheet Algorithm for Stagewise Solvent Extraction'
}

\author{
Ralph A. Leonard and Monica C. Regalbuto
}

Chemical Technology Division

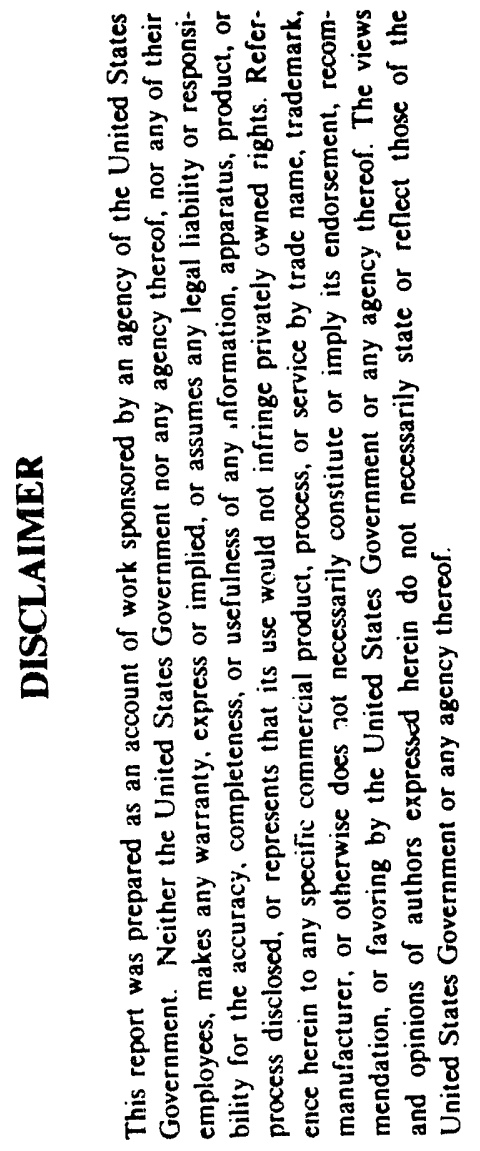

Argonne National Laboratory

Argonne, IL 60439

January 1993

1 Work supported by the U.S. Department of Energy, Nuclear Energy Research and Development Program, under Contract W-31-109-Eng-38. 


\title{
A Spreadsheet Algorithm for Stagewise Solvent Extraction
}

\author{
Ralph A. Leonard and Monica C. Regalbuto
}

Chemical Technology Division, Argonne National Laboratory, Argonne, IL 60439

The material balance and equilibrium equations for solvent extraction processes have been combined with computer spreadsheets in a new way so that models for very complex multicomponent multistage operations can be setup and used easily. A part of the novelty is the way in which the problem is organized in the spreadsheet. In addition, to facilitate spreadsheet setup, a new calculational procedure has been developed. The resulting Spreadsheet Algorithm for Stagewise Solvent Extraction (SASSE) can be used with either IBM or Macintosh personal computers as a simple yet powerful tool for analyzing solvent extraction flowsheets.

\section{Introduction}

The objective of this work was to develop a very general procedure for doing solvent extraction calculations using electronic spreadsheets (worksheets) since they are now widely available on both IBM and Apple Macintosh personal computers. The desired method was to be easy to set up and use, yet able to handle very complex multistage operations with many components. In addition, the method should be suitable for stagewise solvent extraction equipment such as the mixer settler (Davis and Jennings, 1961, Treybal, 1963, Bebbington, 1976) and the centrifugal contactor (Davis and Jennings, 1961, Bernstein et al., 1973, Bebbington, 1976, Leonard et al., 1980, Leonard 1988, Leonard et al., 1992).

Some of the more successful procedures for doing solvent extraction calculations have been McCabe-Thiele diagrams, algebraic solutions, and computer-language codes. McCabe-Thiele diagrams have long been the standard method for evaluating solvent extraction flowsheets (Haas, 1961, Walas, 1988). While these diagrams are very general, they require a lot of time to use as they must be done by hand, they do not allow for backmixing effects, and they can only handle one component at a time. Algebraic solutions have been developed for a number of cases (Alders, 1959). Typically, these solutions handle, at most, two 
sections (extraction/scrub) and assume that the distribution ratio is constant. With more sections, variable distribution coefficients, additional feeds within a section, or backmixing, the algebra becomes very complex and generally not worth the effort. Some computer language codes, for example, the SEPHIS code written in FORTRAN (Groenier, 1972), have been developed over the past 20 years. However, they are generally cumbersome to use, hard to modify, and typically are written for specific components, acids, and solvents.

In addition to these procedures for doing solvent extraction calculations with the drawbacks that they represent, a procedure for using computer spreadsheets has been reported in the literature (Leonard, 1987). In that work, a spreadsheet design was shown that was easy to use and allows a different distribution ratio for each stage. However, that design has several limitations. It can do only one component at a time, does not allow for other-phase carryover (backmixing), does not allow liquid streams into or out of the process within a process section, requires many spreadsheet rows to reach steady state (the exact number of rows determined by a trial-and-error procedure), and assumes an extraction efficiency of $100 \%$. While $100 \%$ extraction efficiency is a reasonable assumption for a centrifugal contactor, it is a poor assumption for a mixer settler.

Building on this prior work, a Spreadsheet Algorithm for Stagewise Solvent Extraction (SASSE) has been under development for some ime. It was continually being refined to meet the requirements of specific solvent extraction flowsheets. An outline of the developing SASSE worksheet was given in a recent paper on the modeling of solvent extraction in columns (Leonard, 1992). The final SASSE worksheet, presented in detail here, is a very general form that is surprisingly easy to use. It applies not only to solvent extraction in columns but also to solvent extraction in stagewise units such as mixer settlers and centrifugal contactors.

The novel feature of the SASSE worksheet is that, by proper organization of the worksheet, the earlier calculational limitations can all be eliminated. A second novel feature is the develorment of a new calculational procedure called the Reduction Thomas algorithm. This algorithm makes for a compact worksheet; it is essential if the worksheet does not do matrix inversions. In this SASSE worksheet, one can specify at every stage (1) an external feed for both phases, (2) a fractional external effluent for both phases, (3) sidestream sampling 
for both phases, (4) other-phase carryover (backmixing) in both phases, and (5) an extraction efficiency. Because of the worksheet structure, many components can be analyzed at the same time. For each component, a different distribution ratio can be specified at every stage. These ratios can be calculated from the component concentrations in a stage if this relationship is known.

This paper is divided into two parts. First, the material balance and equilibrium equations are developed to get the underlying spreadsheet algorithms as well as the Reduction Thomas algorithm. Second, the worksheet organization is presented by which these algorithms are able to give the powerful features of the new SASSE worksheet.

\section{Algorithm Development}

The algorithms developed here are those required for calculating the flow rates and the component concentrations at each process stage. The concentration algorithms are presented first assuming that the flow rates are known. Then the flow rate algorithms are developed including the Reduction Thomas algorithm, a compact new method for calculating the flow rates without using a bulky cumbersome method such as matrix inversion.

\section{Concentrations}

In the earlier work on the use of electronic worksheets (Leonard, 1987), each process stage was assumed to operate as shown in Figure 1. For this simple case there is only one stream flowing into and out of a stage for each phase. For the more complex case that we consider now, the streams flowing into and out of each stage are as shown in Figure 2. This complex case is like that shown in (Leonard, 1992) except that, in addition to all the other flows into and out of the stage, a sidestream with a known flow rate ( $\mathrm{q}_{\mathrm{s}, \mathrm{a}, \mathrm{i}}$ for the aqueous phase at stage $\mathrm{i}$, $\mathrm{q}_{\mathrm{s}, 0, \mathrm{i}}$ for the organic phase) can be specified for each stage. The other flows for each phase are an external feed to the stage, an interstage feed to the stage with some fraction of other-phase carryover (backmixing), and an interstage effluent from the stage with some fraction of other-phase carryover as well as a fraction of the effluent that leaves the process as an external effluent. To simplify the various equations, the ratio of the organic phase to the aqueous phase in the aqueous-phase interstage flow, $R_{a, i}$, is used. This ratio is given by 


$$
\mathrm{R}_{\mathrm{a}, \mathrm{i}}=\frac{\mathrm{f}_{\mathrm{a}, \mathrm{i}}}{1-\mathrm{f}_{\mathrm{a}, \mathrm{i}}}
$$

Similarly, the ratio of the aqueous phase to the organic phase in the organicphase interstage flow, $R_{0, i}$, is given by

$$
\mathrm{R}_{\mathrm{o}, \mathrm{i}}=\frac{\mathrm{f}_{\mathrm{o}, \mathrm{i}}}{1-\mathrm{f}_{\mathrm{o}, \mathrm{i}}}
$$

Note that there is no other-phase carryover in the efflueni or sampling flows. If this situation occurs, it can be modeled by creating (or increasing) an appropriate effluent or sampling flow for the other phase at the same stage. Since other-phase carryover (backmixing) is dealt with in a rigorous fashion, any amount of otherphase carryover can be modeled. Even though the various other-phase carryovers are shown as minor flows in Figure 2, one or more could have a larger flow rate than the associated major flow.

Solving the component material balance for the complex stage operation shown in Figure 2 gives $n$ equations, one for each of the $n$ stages. If each $y_{i}$ factor is replaced by $D_{i} x_{i}$, which is the assumption of $100 \%$ stage efficiency, the resulting equations can be written as

$$
a_{i} x_{i-1}+b_{i} x_{i}+c_{i} x_{i+1}=k_{i} \quad 1 \leq i \leq n
$$

where

$$
\begin{array}{cc}
a_{1}=0 & \\
a_{i}=-\left(1-f_{e, o, i-1}\right)\left(D_{i-1}+R_{o, i-1}\right) q_{o, i-1}, & 2 \leq i \leq n \\
b_{i}=\left[1+\left(1-f_{e, a, i}\right) D_{i} R_{a, i}\right] q_{a, i}+\left[D_{i}+\left(1-f_{e, o, i}\right) R_{o, i}\right] q_{0, i} & 1 \leq i \leq n \\
+q_{s, a, i}+D_{i} q_{s, o, i}, & 1 \leq i \leq n-1 \\
c_{i}=-\left(1-f_{e, a, i+1}\right)\left(1+D_{i+1} R_{a, i+1}\right) q_{a, i+1}, & \\
c_{n}=0 & 1 \leq i \leq n
\end{array}
$$


Since all of the aqueous phase must be an effluent at stage 1 ,

$$
\mathrm{f}_{\mathrm{e}, \mathrm{a}, 1}=1
$$

Since all of the organic phase must be an effluent at stage $n$,

$$
\mathrm{f}_{\mathrm{e}, \mathrm{o}, \mathrm{n}}=1
$$

Equations 3 through 11 can be applied to any component as long as the appropriate $D_{i}$ values are used. In these equations, $n$ is the number of stages for the complete solvent extraction process being modeled. Organic phase is fed to stage 1 and moves to stage $\mathrm{n}$. While additional organic phase can be fed to any other stage, this is normally not done. Aqueous phase is fed to stage $\mathrm{n}$ and moves toward stage 1. Additional aqueous phase is usually fed at various points within the process. In every case the movement of the aqueous phase is towards stage 1. Because of the general nature of these equations, it is no problem to add, delete, or shift sections within the process by adding, deleting, or shifting an external feed or an external effluent.

Assuming that the flows rates are known (the calculational procedure to get these flows is given in the next section), the concentration profiles for a component can be calculated using Eqs. 3 through 10. These $n$ equations have $n$ unknowns $\left(\mathrm{x}_{\mathrm{i}}, \mathrm{i}=1, \ldots, \mathrm{n}\right)$ that must be determined to get the component concentration of the aqueous phase leaving each stage. Being linear equations, they could be solved by a number of general techniques including matrix inversion, the method of determinants, and various Gauss elimination techniques (Hildebrand, 1956, Hohn, 1958). Because the coefficients for this set of equations form a tridiagonal matrix, see Walas (1988), the Thomas algorithm can also be used, see King (1971) and Carnahan et al. (1969). The Thomas algorithm was chosen for use here because it is a compact solution that fits easily into a worksheet layout. Applying the Thomas algorithm to the set of $\mathrm{n}$ equations gives the following set of recursion formulas:

$$
\begin{aligned}
& \beta_{1}=b_{1} \\
& \gamma_{1}=\frac{k_{1}}{\beta_{1}}
\end{aligned}
$$




$$
\begin{array}{lr}
\beta_{i}=b_{i}-\frac{a_{i} c_{i-1}}{\beta_{i-1}}, & i=2,3, \ldots, n \\
\gamma_{i}=\frac{k_{i}-a_{i} \gamma_{i-1}}{\beta_{i}}, & i=2,3, \ldots, n \\
x_{n}=\gamma_{n} & \\
x_{i}=\gamma_{i}-\frac{c_{i} x_{i+1}}{\beta_{i}}, & i=n-1, n-2, \ldots, 1
\end{array}
$$

which can be used to calculate the aqueous-phase concentration for the specified chemical component in the aqueous phase leaving each stage. Once the aqueousphase concentrations are determined, the organic-phase concentrations can be calculated from the distribution ratio for the component at each stage $i$ using

$$
\mathrm{y}_{\mathrm{i}}=\mathrm{D}_{\mathrm{i}} \mathrm{x}_{\mathrm{i}}
$$

Since this procedure can be applied to each chemical component, this is the desired solution for the component concentration equations.

\section{Flow Rates}

In this section, we develop the general equations for calculating the interstage flow of the aqueous and organic phases, $q_{a, i}$ and $q_{o, i}$, respectively, for each stage $i$ as shown in Figure 2. If the assumption of constant density for both phases at every stage is made, the flow balance equation for the aqueous phase in the general stage $i$ is

$$
\begin{array}{rr}
q_{a, i}=q_{f, a, i}-q_{s, a, i}+\left(1-f_{e, a, i+1}\right) q_{a, i+1}+\left(1-f_{e, o, i-1}\right) R_{o, i-1} q_{o, i-1} \\
-\left(1-f_{e, o, i}\right) R_{o, i} q_{o, i}, & 2 \leq i \leq n-1
\end{array}
$$

At stage 1, there is no stage i-1 so that Eq. 19 becomes

$$
q_{a, 1}=q_{f, a, 1}-q_{s, a, 1}+\left(1-f_{e, a, 2}\right) q_{a, 2}-\left(1-f_{e, 0,1}\right) R_{o, 1} q_{o, 1}
$$

At stage $n$, there is no stage $i+1$ and $f_{e, 0, i}$ becomes 1.0 so that Eq. 19 becomes

$$
q_{a, n}=q_{f, a, n}-q_{s, a, n}+\left(1-f_{e, o, n-1}\right) R_{o, n-1} q_{o, n-1}
$$


The corresponding flow balance equations for the organic phase in the general stage $i$ is

$$
\begin{aligned}
q_{o, i}=q_{f, o, i}-q_{s, o, i}+\left(1-f_{e, o, i-1}\right) q_{o, i-1}+\left(1-f_{e, a, i+1}\right) R_{a, i+1} & q_{a, i+1} \\
-\left(1-f_{e, a, i}\right) R_{a, i} q_{a, i}, & 2 \leq i \leq n-1
\end{aligned}
$$

At stage 1 , there is no stage $\mathrm{i}-1$ and $\mathrm{f}_{\mathrm{e}, \mathrm{a}, \mathrm{i}}$ becomes 1.0 so that Eq. 22 becomes

$$
q_{o, 1}=q_{f, 0,1}-q_{s, 0,1}+\left(1-f_{e, a, 2}\right) R_{a, 2} q_{a, 2}
$$

At stage $n$, there is no stage $i+1$ so that Eq. 22 becomes

$$
q_{o, n}=q_{f, o, n}-q_{s, o, n}+\left(1-f_{e, o, n-1}\right) q_{o, n-1}-\left(1-f_{e, a, n}\right) R_{a, n} q_{a, n}
$$

Equations 19 through 24 characterize the flow of the aqueous and organic phases in any stage of a process that has complex stage operation. Since external feeds can be added to any stage and external effluents can be taken from any stage, all or part of an effluent from one stage can become a feed for a non-adjacent stage. This feature can be used to model process operations such as (1) solvent recycle and (2) special strip sections, for example, a strip section expanded to include its own scrub section (Siddall, 1961).

Equations 19 through 24 represent $2 n$ linear equations that must be solved for the $2 \mathrm{n}$ unknowns $\left(\mathrm{q}_{\mathrm{a}, \mathrm{i}}\right.$ and $\left.\mathrm{q}_{\mathrm{o}, \mathrm{i}}, \mathrm{i}=1, \ldots, \mathrm{n}\right)$. As noted above, a number of general techniques for linear equations can be used including matrix inversion. Because the equation coefficients form a heptadiagonal matrix instead of a tridiagonal matrix, the Thomas algorithm can not be used. However, these $2 \mathrm{n}$ equations in $\mathrm{q}_{\mathrm{a}, \mathrm{i}}$ and $\mathrm{q}_{\mathrm{o}, \mathrm{i}}$ can be reduced to $\mathrm{n}$ equations in $\mathrm{q}_{\mathrm{a}, \mathrm{i}}$ by a reduction algorithm which consists of algebraically decoupling the organic and aqueous flows. The coefficients for this set of $\mathrm{n}$ equations do form a tridiagonal matrix so that the Thomas algorithm can be used. This decoupling procedure and subsequent use of the Thomas algorithm, which we call the "Reduction Thomas algorithm," is derived as follows.

To derive the Reduction Thomas algorithm, one first combines Eqs. 19 and 22 to eliminate $\mathrm{q}_{0, \mathrm{i}-1}$ and get 


$$
\begin{gathered}
q_{o, i}\left[\left(1-f_{e, o, i}\right) R_{o, i}-R_{o, i-1}\right]+q_{a, i}\left[1-\left(1-f_{e, a, i}\right) R_{a, i} R_{o, i-1}\right] \\
+q_{a, i+1}\left(R_{a, i+1} R_{o, i-1}-1\right)\left(1-f_{e, a, i+1}\right) \\
=q_{f, a, i}-q_{s, a, i}-R_{o, i-1}\left(q_{f, o, i}-q_{s, o, i}\right)
\end{gathered}
$$

Next one combines Eqs. 19 and 22 to eliminate $\mathrm{q}_{0, \mathrm{i}}$ and get

$$
\begin{aligned}
q_{o, i-1}\left(1-f_{e, o, i-1}\right) & {\left[R_{o, i-1}-\left(1-f_{e, o, i}\right) R_{o, i}\right]+q_{a, i}\left[\left(1-f_{e, a, i}\right)\left(1-f_{e, o, i}\right) R_{a, i} R_{o, i}-1\right] } \\
& +q_{a, i+1}\left(1-f_{e, a, i+1}\right)\left[1-\left(1-f_{e, o, i}\right) R_{o, i} R_{a, i+1}\right] \\
& =\left(1-f_{e, o, i}\right) R_{o, i}\left(q_{f, o, i}-q_{s, o, i}\right)-q_{f, a, i}+q_{s, a, i}
\end{aligned}
$$

Finally, one takes Eq. 25 for stage i and writes it for stage i-1. The resulting equation is combined with Eq. 26 for stage $i$ to eliminate $q_{0, i-1}$, the only remaining organic-phase flow term in the combined equation. After some algebraic manipulations, the final equation for stage i can be written in terms of the aqueous-phase flows as

$$
A_{i} q_{a, i-1}+B_{i} q_{a, i}+C_{i} q_{a, i+1}=K_{i}
$$

where the aqueous-flow coefficients $A_{i}, B_{i}$, and $C_{i}$ and the constant term $K_{i}$ for the $\mathrm{n}$ stages are given by

$$
\begin{gathered}
A_{1}=0 \\
A_{2}=\left(1-f_{e, o, 1}\right)\left[R_{o, 1}-\left(1-f_{e, o, 2}\right) R_{o, 2}\right] \\
A_{i}=\left(1-f_{e, o, i-1}\right)\left[R_{o, i-1}-\left(1-f_{e, o, i}\right) R_{o, i}\right] \\
{\left[1-\left(1-f_{e, a, i-1}\right) R_{a, i-1} R_{o, i-2}\right],} \\
B_{1}=1 \\
B_{2}=\left(1-f_{e, o, 1}\right)\left\{\left[\left(1-f_{e, 0,2}\right) R_{o, 2}-R_{o, 1}\right]\left(1-f_{e, a, 2}\right)\right. \\
\left.-\left[\left(1-f_{e, a, 2}\right)\left(1-f_{e, o, 2}\right) R_{a, 2} R_{o, 2}-1\right] R_{o, 1}\right\} \\
B_{i}=\left(1-f_{e, o, i-1}\right)\left[\left(1-f_{e, o, i}\right) R_{o, i}-R_{o, i-1}\right]\left(1-f_{e, a, i}\right)\left(1-R_{a, i} R_{o, i-2}\right) \\
-\left[\left(1-f_{e, a, i}\right)\left(1-f_{e, o, i}\right) R_{a, i} R_{o, i}-1\right]\left[\left(1-f_{e, o, i-1}\right) R_{o, i-1}-R_{o, i-2}\right], \\
i=3, \ldots, n \\
C_{1}=\left(1-f_{e, a, 2}\right)\left[\left(1-f_{e, 0,1}\right) R_{o, 1} R_{a, 2}-1\right] \\
C_{2}=\left(1-f_{e, a, 3}\right)\left[\left(1-f_{e, o, 2}\right) R_{o, 2} R_{a, 3}-1\right]\left(1-f_{e, o, 1}\right) R_{o, 1}
\end{gathered}
$$




$$
\begin{gathered}
C_{i}=\left(1-f_{e, a, i+1}\right)\left[\left(1-f_{e, o, i}\right) R_{o, i} R_{a, i+1}-1\right]\left[\left(1-f_{e, o, i-1}\right) R_{o, i-1}-R_{o, i-2}\right], \\
i=3, \ldots, n-1 \\
C_{n}=0 \\
K_{1}=q_{f, a, 1}-q_{s, a, 1}-\left(1-f_{e, o, 1}\right) R_{o, 1}\left(q_{f, o, 1}-q_{s, o, 1}\right) \\
K_{2}=\left(1-f_{e, o, 1}\right)\left\{\left[R_{o, 1}-\left(1-f_{e, o, 2}\right) R_{o, 2}\right]\left(q_{f, a, 1}-q_{s, a, 1}\right)\right. \\
\left.-\left[\left(1-f_{e, o, 2}\right) R_{o, 2}\left(q_{f, o, 2}-q_{s, 0,2}\right)-\left(q_{f, a, 2}-q_{s, a, 2}\right)\right] R_{o, 1}\right\} \\
\quad K_{i}=\left(1-f_{e, o, i-1}\right)\left[R_{o, i-1}-\left(1-f_{e, o, i}\right) R_{o, i}\right] \\
{\left[\left(q_{f, a, i-1}-q_{s, a, i-1}\right)-\left(q_{f, o, i-1}-q_{s, o, i-1}\right) R_{o, i-2}\right]} \\
-\left[\left(1-f_{e, o, i}\right) R_{o, i}\left(q_{f, o, i}-q_{s, o, i}\right)-\left(q_{f, a, i}-q_{s, a, i}\right)\right] \\
{\left[\left(1-f_{e, o, i-1}\right) R_{o, i-1}-R_{o, i-2}\right], \quad i=3, \ldots, n}
\end{gathered}
$$

The $\mathrm{n}$ equations contained in Eq. 27 can be solved for the $\mathrm{n} \mathrm{q}_{\mathrm{a}, \mathrm{i}}$ 's by using the Thomas algorithm, Eqs. 12 through 17, where $\mathrm{x}_{\mathrm{i}}, \mathrm{a}_{\mathrm{i}}, \mathrm{b}_{\mathrm{i}}, \mathrm{c}_{\mathrm{i}}$, and $\mathrm{k}_{\mathrm{i}}$ are replaced by $\mathrm{q}_{\mathrm{a}, \mathrm{i}}, \mathrm{A}_{\mathrm{i}}, \mathrm{B}_{\mathrm{i}}, \mathrm{C}_{\mathrm{i}}$, and $\mathrm{K}_{\mathrm{i}}$, respectively. Once the aqueous-phase flow rates are determined at each stage, the organic-phase flow rates can be calculated from Eq. 25 .

The residence time of the aqueous phase in stage i can be calculated as

$$
t_{a, i}=\frac{v_{a, i}}{q_{a, i}+q_{s, a, i}+\left(1-f_{e, o, i}\right) R_{o, i} q_{o, i}}
$$

if the volume of aqueous phase in the stage, $\mathrm{V}_{\mathrm{a}, \mathrm{i}}$, is known. The residence time of the organic phase in stage $i$ can be calculated as

$$
\mathrm{t}_{\mathrm{o}, \mathrm{i}}=\frac{\mathrm{v}_{\mathrm{o}, \mathrm{i}}}{\mathrm{q}_{\mathrm{o}, \mathrm{i}}+\mathrm{q}_{\mathrm{s}, \mathrm{o}, \mathrm{i}}+\left(1-\mathrm{f}_{\mathrm{e}, \mathrm{a}, \mathrm{i}}\right) \mathrm{R}_{\mathrm{a}, \mathrm{i}} \mathrm{q}_{\mathrm{a}, \mathrm{i}}}
$$

if the volume of organic phase in the stage, $\mathrm{V}_{0, \mathrm{i}}$, is known.

\section{Extraction Efficiency}

Up to this point, it has been assumed that the extraction efficiency of each stage is $100 \%$. To emphasize this, Eq. 18 is rewritten here with an asterisk on each concentration 


$$
\mathrm{D}_{\mathrm{i}}=\frac{\mathrm{y}_{\mathrm{i}}^{*}}{\mathrm{x}_{\mathrm{i}}^{*}}
$$

to indicate that the two phases are equilibrated. When the exiraction efficiency is less than $100 \%$, an effective distribution ratio is written as

$$
\mathrm{D}_{\mathrm{eff}, \mathrm{i}}=\frac{\mathrm{y}_{\mathrm{i}}}{\mathrm{x}_{\mathrm{i}}}
$$

where $x_{i}$ and $y_{i}$ are the actual concentrations of the aqueous and organic phases, respectively, leaving stage $i$. With a fractional extraction efficiency defined as

$$
E_{i}=\frac{x_{i+1}-x_{i}}{x_{i+1}-x_{i}^{*}}
$$

it has been shown by Leonard (1987) that

$$
D_{e f f, i}=\frac{D_{i} E_{i}+R_{i} D_{i} S_{i}\left(1-E_{i}\right)+S_{i}\left(1-E_{i}\right)}{1-R_{i} D_{i}\left(1-E_{i}\right)}
$$

where $R_{i}$ is the ratio of organic to aqueous phase flowing through stage $i$ and $S_{i}$ is the ratio of the component concentration in the organic phase entering into stage $i$ from stage i-1 to the component concentration in the aqueous phase leaving stage i. For simple stage operation, the $R_{i}$ and $S_{i}$ equations given by Leonard (1987) can be used. For complex stage operation, $R_{i}$ is given by

$$
R_{i}=\frac{q_{o, i}+q_{s, 0, i}+\left(1-f_{e, a, i}\right) R_{a, i} q_{a, i}}{q_{a, i}+q_{s, a, i}+\left(1-f_{e, o, i}\right) R_{o, i} q_{o, i}}
$$

and $\mathrm{S}_{\mathrm{i}}$ is given by

$$
\mathrm{S}_{\mathrm{i}}=\frac{\mathrm{y}_{\mathrm{i}-1, \mathrm{avg}}}{\mathrm{x}_{\mathrm{i}}}
$$

where $y_{i-1, a v g}$ is the average component concentration in the organic phase entering -tage i given by

$$
y_{i-1, a v g}=\frac{\left(1-f_{e, 0, i-1}\right) q_{o, i-1} y_{i-1}+q_{f, o, i} y_{f, i}+\left(1-f_{e, a, i+1}\right) R_{a, i+1} q_{a, i+1} y_{i+1}}{\left(1-f_{e, 0, i-1}\right) q_{0, i-1}+q_{f, o, i}+\left(1-f_{e, a, i+1}\right) R_{a, i+1} q_{a, i+1}}
$$


Equations 47 and 48 reduce to the simple stage equations given by Leonard (1987) when all the $f_{e, a, i}$ 's, $f_{e, o, i}$ 's, $q_{f, a, i}$ 's, $q_{f, o, i}$ 's, $q_{s, a, i}$ 's, $q_{s, o, i}$ 's, $R_{a, i}$ 's, and $R_{0, i}$ 's are zero. Since Eq. 10 applies at stage 1 and there is no stage $0, R_{i}$ and $y_{i-1, \text { avg }}$ for stage 1 are given by

$$
R_{1}=\frac{q_{0,1}+q_{s, 0,1}}{q_{a, 1}+q_{s, a, 1}+\left(1-f_{e, o, 1}\right) R_{o, 1} q_{o, 1}}
$$

and

$$
y_{0, a v g}=\frac{q_{f, o, 1} y_{f, 1}+\left(1-f_{e, a, 2}\right) R_{a, 2} q_{a, 2} y_{2}}{q_{f, o, 1}+\left(1-f_{e, a, 2}\right) R_{a, 2} q_{a, 2}}
$$

Since Eq. 11 applies at stage $n$ and there is no stage $n+1, R_{i}$ and $y_{i-1, \text { avg }}$ for stage $n$ are given by

$$
R_{n}=\frac{q_{o, n}+q_{s, 0, n}+\left(1-f_{e, a, n}\right) R_{a, n} q_{a, n}}{q_{a, n}+q_{s, a, n}}
$$

and

$$
y_{n-1, a v g}=\frac{\left(1-f_{e, 0, n-1}\right) q_{o, n-1} y_{n-1}+q_{f, o, n} y_{f, n}}{\left(1-f_{e, 0, n-1}\right) q_{o, n-1}+q_{f, o, n}}
$$

Equations 46 through 53 can be used to calculate the $D_{\text {eff, } i}$ values for any stage $i$ $(\mathrm{i}=1, \ldots, \mathrm{n})$ in a complex stage operation.

Thus, when the extraction efficiency is less than $100 \%$, that is, $\mathrm{E}_{\mathrm{i}}$ is less than 1.0 , the $D_{i}$ values in $a_{i}, b_{i}$ and $c_{i}$ coefficients of Eq. 3 are replaced by $D_{e f f, i}$ values. Since $D_{e f f, i}$ depends not only on $D_{i}, E_{i}$, and $R_{i}$ but also on $S_{i}$, these material balance equations now require an iterative solution. While such a solution is easy to carry out using an electronic worksheet, it does increase the time to recalculate the worksheet.

\section{Worksheet Organization}

We now present a new way to organize electronic worksheets so that the detailed model given above can be implemented easily. The basic structure is to have the variable name in the first column, the values of this variable in stages 1 through $\mathrm{n}$ appear in columns 2 through $\mathrm{n}+1$, and a short description of the 
variable, especially variables that are supplied by the user, in column $n+2$. Each variable would have its own row. For example, the variable $f_{e, o, i}$, which might be named FEOI, would have its own row. Appropriate values would be supplied by the user for all $n$ stages starting at column 2 . In column $n+2$, a note might be added such as "Fraction of exiting $\mathrm{O}$ taken as an effluent from each stage."

Keeping this basic worksheet structure of information for a specific variable in a specific worksheet row and information for a specific stage in a specific worksheet column, the worksheet rows are arranged into sections as shown in Figure 3. We now discuss each of these sections: general notes, user specified parameters, $D$ values, summary of calculations, flow balance calculations, and mass balance equations. After this discussion of the various worksheet sections, good general practices are given for setting up such a worksheet.

\section{General Notes}

The top section of the SASSE worksheet contains general notes. These notes could give information for user reference as well as variables and limits that need to be input with the value used displayed in a spreadsheet cell. Typical notes might include creation and revision date, number of sections and stages, number of components, and component names as well as other information that will be needed to specify the worksheet.

\section{User Specified Parameters}

Except for the $\mathrm{D}$ values, all of the user specified parameters for each stage are entered here. The parameters that must be specified are the stage numbers (i), $E_{i}, f_{a, i}, f_{o, i}, q_{s, a, i}, q_{s, o, i}, f_{e, a, i}, f_{e, o, i}, q_{f, a, i}, q_{f, o, i}$, and $x_{f, i}$ and $y_{f, i}$ for each of the components at each stage where there is an aqueous or organic feed to the proces?. Besides these required rows, other rows could give section identification and feed and effluent code iames.

By entering this input data following the basic row structure, all of the user specified parameters are in one place where they can be easily accessed as they are needed in subsequent worksheet calculations. 


\section{Values}

User specified D values are entered in this section of the worksheet again following the basic row structure. If the $D$ values for a component are known for each stage, a single row can be used to specify the $\mathrm{D}$ values for the component. If the $\mathrm{D}$ values for a component must be calculated based on the concentration of one or more components, several rows may be required.

\section{Summary of Calculations}

After the two worksheet sections where the user specifies the various process parameters, a summary of the calculations is given. While this section is not essential, it is convenient to have all the results summarized in one place. These results would include the external effluent flows from each stage $\left(q_{e, a, i}\right.$ and $\mathrm{q}_{\mathrm{e}, \mathrm{o}, \mathrm{i}}$ ) and the concentrations in the aqueous and organic phase exiting each stage $\left(x_{i}\right.$ and $y_{i}$ ) for each component.

\section{Flow Balance Calculations}

The flow rates for the aqueous and organic phases exiting from each stage $\left(q_{a, i}\right.$ and $q_{o, i}$, respectively) are calculated in this section of the SASSE worksheet. The first several rows are dedicated to checking selected input values such as $f_{a, i}$, $f_{o, i}, q_{s, a, i}, q_{s, o, i}, f_{e, a, i}$, and $f_{e, o, i}$ and seeing that their input values are not inappropriate. In particular, $\mathrm{f}_{\mathrm{o}, \mathrm{i}}$ and $\mathrm{f}_{\mathrm{e}, 0, \mathrm{i}}$ must be equal to or greater than some minimum value or else round-off error becomes important when one uses the recursive equations of the Thomas algorithm. (For the Excel worksheet, which works with 15 significant digits, the minimum value is $1 \times 10^{-9}$. This minimum value is expected to be somewhat dependent on computer software.) Then $R_{a, i}$ and $R_{o, i}$ values are calculated from $f_{a, i}$ and $f_{o, i}$, respectively.

After these preliminary calculations, the values for $A_{i}, B_{i}, C_{i}$, and $K_{i}$ are calculated for each stage as given by Eqs. 28 through 40 . These coefficients are then used to get the $\beta_{\mathrm{i}}$ and $\gamma_{\mathrm{i}}$ values of the Thomas algorithm as given by Eqs. 12 through 15 from which the $\mathrm{q}_{\mathrm{a}, \mathrm{i}}$ values for each stage are calculated. The $\mathrm{q}_{0, \mathrm{i}}$ values are calculated from these aqueous flow rates and then, using both sets of flow rates, the $R_{i}$ values are also calculated. Once these flow rates have been determined, the concentration of each component in each stage is calculated in the section called mass balance calculations. 


\section{Mass Balance Calculations}

The concentration of each component in the aqueous and organic phases exiting each stage $\left(x_{i}\right.$ and $y_{i}$, respectively) are calculated in this section of the SASSE worksheet. The arrangement of the worksheet for the first component is given here. This same arrangement is then repeated for each component. The first four rows bring in the values for $D_{i}, x_{i}, y_{i-1}$, and $y_{i+1}$ and checks to see that these values are not unreasonable. As was done in the above sections, the row format is used for each variable with its name in column 1 , the stage 1 value in column 2 , the stage 2 value in column 3 , etc. Using these values as well as others taken directly from previous sections, the $S_{i}$ and $D_{\text {eff,i }}$ values are calculated.

After these preliminary calculations, the values for $a_{i}, b_{i}, c_{i}$, and $k_{i}$ are calculated for each tage as given by Eqs. 4 through 9 . These coefficients are then used to get the $\beta_{\mathrm{i}}$ and $\gamma_{\mathrm{i}}$ values of the Thomas algorithm as given by Eqs. 12 through 15 from which the $x_{i}$ values for each stage are calculated. The $y_{i}$ values are calculated from the aqueous-phase concentrations using Eq. 44.

\section{Naming Conventions and General Practices}

One useful naming convention is to identify a variable for a specific component with its component number " $j$ " by adding the suffix " $j$ " at the end of each variable name. Thus, the aqueous feed concentration for the second component might be written as "xf_2" in the worksheet. By doing this, it is easy to add components.

A good general practice is setting up worksheets where calculations are so dependent on one another is to set up error traps at key points. For example, when an important variable is taken from one part of the worksheet to be used in another part of the worksheet, it can be checked to see if there is an error message in the cell or if the variable has too high or too low a value. If any of these conditions occur, the trap would supply an appropriate alternative value. These traps are particularly important for iterative solutions where extreme values may need to be suppressed until the calculations start to converge on an appropriate set of values. 
Another good general practice is to mark those cells for which the user must specify the value, for example, a bottom border can be added to such worksheet cells.

\section{Discussion}

The SASSE algorithm presented here is a new way to set up computer spreadsheets so that they can be used to model a very wide range of stagewise solvent $\epsilon$ itraction processes at steady-state operation. The new Reduced Thomas algorithm allows essentially any electronic worksheet to be used and keeps the worksheet compact. By focusing on a single stage, a high level of process complexity can be introduced while keeping the resulting equations manageable. While we have only run the SASSE algorithm using Microsoft Excel on the Macintosh and IBM personal computers, there is no reason why other spreadsheets could not be used. This procedure could also be carried out using a programming language such as BASIC, FORTRAN, C, or Pascal, although a lot of the ease of use will be lost.

It is possible to use a spreadsheet macro to setup the SASSE worksheet. This may be a good idea if one will be setting up the SASSE worksheet often for different numbers of stages and components. However, the worksheet is not difficult to set up from scratch and even easier to modify if one starts with an earlier worksheet. In particular, as can be seen lcoking at the equations above, they are the same for all stages except stages 1 and $n$ and, for some coefficients of the flow equations, stage 2 .

If a macro is written to generate the SASSE worksheet, we recommend that the macro be divided into two parts. The first part would present a dialog box to get the number of sections and number of components, create the General Notes part of the SASSE worksheet, and stop. The user would then specify the required information. The second part of the macro would use this information to generate the rest of the SASSE worksheet with the appropriate number of stages and components and the appropriate number of rows for the $\mathrm{D}$ value of each component.

The SASSE worksheet is currently being used to calculate stage-to-stage concentration profiles for the TRUEX process. TRUEX is a solvent extraction process for removing and recovering long-lived radioactive elements such as 
americium and plutonium from nuclear wastes, see Vandegrift et al. (1984), Leonard et al. (1985), Horwitz and Schulz (1986), and Schulz and Horwitz (1988), using an extractant, octyl(phenyl)-N,N-diisobutylcarbamoylmethylphosphine oxide (CMPO), a phase modifier, tributyl phosphate (TBP), and a diluent such as normal dodecane ( $\mathrm{nDD}$ ), tetrachloroethylene (TCE), or carbon tetrachloride $\left(\mathrm{CCl}_{4}\right)$. The SASSE worksheet was applied to the 14-stage TRUEX process shown

in Figure 4. With the user specified parameters in Table 1 (note that the component concentrations are specified for the organic feed, that is, there is no solvent recycle for this case) and the $\mathrm{D}$ values in Table 2 , the external flow rates and the concentration profiles were calculated using a SASSE worksheet and the results are summarized in Table 3 . In this case, the model points out the need for more stages in the first strip section so that the americium does not end up in the aqueous effluent from the second strip which is the plutonium strip section.

Computer times were measured for the SASSE worksheet using the TRUEX flowsheet shown in Figure 4. Calculations were done on an Apple Macintosh IIci computer with an Apple color monitor set at 256 colors, Apple System 7.0, and Microsoft Excel 3.0. The worksheet had 5 components with constant $D$ values and 14 stages. With $100 \%$ extraction efficiency, recalculation times were 7 seconds with no organic-phase recycle and 23 seconds with organicphase recycle which required 9 iterations. With $80 \%$ extraction efficiency (essentially the same timing results can be obtained for any extraction efficiency less than 100\%), recalculation times were 96 seconds for 20 iterations and 186 seconds for 40 iterations. In this example, the convergence was slowest for the concentration of the aqueous phase at stage 1 of a two-stage scrub section. This concentration was within $5 \%$ of its final converged value after 20 iterations and was essentially at a steady-state value after 40 iterations. The use of organicphase recycle has no effect on the recalculation time when extraction was less than $100 \%$.

The SASSE worksheet gives a steady-state solution for the solvent extraction process. This makes for a compact worksheet that has short recalculation times, can handle many components, and can accommodate complex multistage operations.

The SASSE algorithm given here assumes that the density of each phase is constant so that the flow balance equation can be used as presented. Thus, flow 
rates are in volumetric units such as $\mathrm{L} / \mathrm{min}$ (or $\mathrm{L} / \mathrm{h}$ ) and the concentration are in units of moles of component per liter of solution, that is, in molar (M) units. If the assumption of constant density is not a good one, then one should work with concentrations in moles of component per kilogram of solvent, that is, in molal (m) units. All the equations given here apply if one also gives the flow rates in kilograms of solvent per minute (or kilograms of solvent per hour) and the D values are calculated using concentrations in molal units. For the TRUEX process, the solvent in the aqueous phase is pure water. In the organic phase, the solvent is the extractant and any phase modifier (TBP in the example) along with the diluent in which they are dissolved.

The SASSE worksheet has been successfully used to model the TRUEX process. It can be applied to a wide variety of stagewise solvent extraction processes with many components, significant other-phase carryover, feeds to any stage, effluents from any stage, and effluent recycle from any stage to any other stage. Thus it can be applied to stagewise units like centrifugal contactors which have essentially $100 \%$ extraction efficiency. However, because an extraction efficiency less than $100 \%$ can be specified, the SASSE worksheet can be applied to other stagewise units like mixer settlers with extraction efficiencies of less than $100 \%$, typically in the 50 to $80 \%$ range. Final'y, using SASSE, a new column model has also been developed. It treats solvent extraction in columns as a stagewise process with a high degree of backmixing of the continuous phase between stages, see Leonard et al. (1990). The column model allows one to specify little or no backmixing between columns, making it easy to evaluate the advantages of having separate extraction and scrub columns.

Since the user can specify the $D_{i}$ value for each component at each stage, the SASSE worksheet is very general regarding the components it can handle. One could even guess the D values for a component, run the SASSE worksheet, and re-estimate the $\mathrm{D}$ values based on the calculated stage composition for the components Operating the SASSE worksheet in this mode, even very rudimentary $D$ value information can be used and the importance of knowing the $\mathrm{D}$ value or values more accurately can be determined.

While this work has focused on good ways for solving complex solvent extraction problems using spreadsheets on personal computers, the general techniques involved should work when using computer worksheets for any 
stagewise countercurrent separation process. The key is to focus on a single stage looking at both the material balance and the separating principle, for example, liquid-liquid equilibrium for solvent extraction. For non-isothermal processes such as distillation, one would also need to include an energy balance for each stage.

\section{Conclusions}

The SASSE algorithm is a powerful new tool for evaluating solvent extraction processes with many components, component interactions, complex component equilibria, feeds to any stage, effluents from any stage, sidestream sampling from any stage, high backmixing (other-phase carryover), effluent recycle, and extraction efficiencies less than $100 \%$. This is accomplished by efficiently organizing a worksheet so that it is not only practical but also very general. The new Reduction Thomas algorithm keeps the worksheet compact and allows one to use essentially any electr jnic worksheet. The overall result is an efficient way for evaluating complex solvent extraction processes that is easy to use and can be set up quickly on a personal computer.

\section{Acknowledgment}

This work was supported by the U. S. Department of Energy under Contract W-31-109-Eng-38.

\section{Notation}

$a_{i} \quad$ coefficient for $x_{i-1}$ of the component material balance equation at stage i

$A_{i} \quad$ coefficient for $q_{a, i-1}$ of the flow balance equation at stage $i$

$b_{i} \quad$ coefficient for $x_{i}$ of the component material balance equation at stage $\mathrm{i}$

$B_{i} \quad$ coefficient for $q_{a, i}$ of the flow balance equation at stage $i$

$c_{i} \quad$ coefficient for $x_{i+1}$ of the component material balance equation at stage $\mathrm{i}$

$\mathrm{C}_{\mathrm{i}} \quad$ coefficient for $\mathrm{q}_{a, i+1}$ of the flow balance equation at stage $\mathrm{i}$

$D_{i} \quad$ distribution ratio for a component at stage $i$ given by $y_{i} / x_{i}$ 
$E_{i} \quad$ fractional extraction efficiency for stage $i$

$f_{a, i} \quad$ fraction of organic-phase carryover in the interstage flow of the aqueous phase from stage $i$

$f_{o, i} \quad$ fraction of aqueous-phase carryover in the interstage flow of the organic phase from stage $i$

$\mathrm{k}_{\mathrm{i}} \quad$ constant term for the component material balance equation at stage $\mathrm{i}$

$\mathrm{K}_{\mathrm{i}} \quad$ constant term for the flow balance equation at stage $\mathrm{i}$

$\mathrm{n}$ number of stages for the solvent extraction process being modelled

$q_{a, i} \quad$ volumetric flow rate for aqueous phase leaving stage $i$, does not include $\mathrm{q}_{\mathrm{s}, \mathrm{a}, \mathrm{i}}$, any organic-phase carryover in the aqueous phase, or any aqueous phase carried out with the exiting organic phase

$q_{f, a, i} \quad$ volumetric flow rate for the aqueous feed to stage $i$

$\mathrm{q}_{\mathrm{f}, \mathrm{o}, \mathrm{i}} \quad$ volumetric flow rate for the organic feed to stage $i$

$q_{0, i} \quad$ volumetric flow rate for organic phase leaving stage $i$, does not include $\mathrm{q}_{\mathrm{s}, \mathrm{o}, \mathrm{i}}$, any aqueous-phase carryover in the organic phase, or any organic phase carried out with the exiting aqueous phase

$\mathrm{q}_{\mathrm{s}, \mathrm{a}, \mathrm{i}} \quad$ fixed sampling rate of the aqueous phase leaving stage $\mathrm{i}$

$\mathrm{q}_{\mathrm{s}, \mathrm{o}, \mathrm{i}} \quad$ fixed sampling rate of the organic phase leaving stage $\mathrm{i}$

$R_{a, i} \quad$ ratio of organic phase to aqueous phase in the aqueous interstage flow from stage $i$

$R_{i} \quad$ ratio of organic-phase flow out of stage $i$ to aqueous-phase flow out of stage i

$R_{0, i} \quad$ ratio of aqueous phase to organic phase in the organic interstage flow from stage $\mathrm{i}$

$\mathrm{S}_{\mathrm{i}}$ ratio of the component concentration in the organic phase from stage $\mathrm{i}-1$ to the component concentration in the aqueous phase from stage $\mathrm{i}$

$t_{a, i} \quad$ residence time of the aqueous phase in stage $i$

$t_{0, i} \quad$ residence time of the organic phase in stage $i$ 
$\mathrm{V}_{\mathrm{a}, \mathrm{i}} \quad$ volume of the aqueous phase in stage $\mathrm{i}$

$\mathrm{V}_{0, \mathrm{i}} \quad$ volume of the organic phase in stage $\mathrm{i}$

$x_{f, i} \quad$ component concentration per unit volume in aqueous feed to stage $i$

$\mathrm{x}_{\mathrm{i}} \quad$ actual component concentration per unit volume in aqueous phase leaving stage $\mathrm{i}$

$\mathrm{x}_{\mathrm{i}}^{*} \quad$ component concentration per unit volume in aqueous phase leaving stage $i$ when the two phases are fully equilibrated

$y_{f, i} \quad$ component concentration per unit volume in organic feed to stage $i$

$\mathrm{y}_{\mathrm{i}} \quad$ actual component concentration per unit volume in organic phase leaving stage $\mathrm{i}$

$y_{i}^{*} \quad$ component concentration per unit volume in organic phase leaving stage $i$ when the two phases are fully equilibrated

$y_{i, a v g}$ average component concentration per unit volume in organic phase leaving stage $\mathrm{i}$

\section{Subscripts}

a aqueous phase

e effluent from a stage, that is, that part of a phase exiting a stage that does not go to the next stage

f feed to a stage

i stage number

j component number

o organic phase

\section{Greek letters}

$\beta_{\mathrm{i}}$ term in the first recursion formula of the Thomas algorithm for stage $\mathrm{i}$

$\gamma_{i}$ term in the second recursion formula of the Thomas algorithm for stage $i$ 


\section{Literature Cited}

Alders, L., Liquid-Liquid Extraction, 2nd ed., Elsevier Publishing, Amsterdam (1959).

Beb'ington, W. P., "The Reprocessing of Nuclear Fuels," Scientific American, 235, no. 6 (December), 30-41 (1976).

Bernstein, G. J., D. E. Grosvenor, J. F. Lenc, and N. M. Levitz, "A High-Capacity Annular Centrifugal Contactor," Nucl. Technol., 20, 200-202 (1973).

Carnahan, B., H. A. Luther, and J. O. Wilkes, Applied Numerical Methods, Wiley, New York (1969).

Davis, M. W. Jr., and A. S. Jennings, "Equipment for Processing by Solvent Extraction," Chapter VII in Chemical Processing of Reactor Fuels, J. F. Flagg, editor, Academic Press, New York, 271-303 (1961).

Groenier, W. S., "Calculation of the Transient Behavior of a Dilute-PUREX Solvent Extraction Process Having Application to the Reprocessing of LMFBR Fuels," Oak Ridge National Laboratory, ORNL-4746 (1972).

Haas, W. O. Jr., "Solvent Extraction: General Principles," Chapter IV in Chemical Processing of Reactor Fuels, J. F. Flagg, editor, Academic Press, New York, 125-198 (1961).

Hildebrand, F. B., Introduction to Numerical Analysis, McGraw-Hill, New York (1969).

Hohn, F. E., Elementary Matrix Algebra, Macmillan, New York (1958).

Horwitz, E. P., and W. W. Schulz, "Application of the TRUEX Process to the Decontamination of Nuclear Waste Streams," International Solvent Extraction Conference, Munich, Federal Republic of Germany, September 11-16, 1986, Vol. I, 81-89 (1986).

King, C. J., Separation Processes, McGraw-Hill, New York (1971).

Leonard, R. A., G. J. Bernstein, A. A. Ziegler, and R. H. Pelto, "Annular Centrifugal Contactors for Solvent Extraction," Sep. Sci. Technol., 15, 925943 (1980).

Leonard, R. A., G. F. Vandegrift, D. G. Kalina, D. F. Fischer, R. W. Bane, L. Burris, E. P. Horwitz, R. Chiarizia, and H. Diamond, "The Extraction and Recovery of Plutonium and Americium from Nitric Acid Waste Solutions by the TRUEX Process--Continuing Development Studies," Argonne National Laboratory, ANL-85-45 (1985).

Leonard, R. A., "Use of Electronic Worksheets for Calculations of Stagewise Solvent Extraction Processes," Sep. Sci. Technol., 22, 535-556 (1987). 
Leonard, R. A., "Recent Advances in Centrifugal Contactor Design," Sep. Sci. Technol., 23, 1473-1487 (1988).

Leonard, R. A., M. C. Regalbuto, D. B. Chamberlain, and G. F. Vandegrift, "A New Model for Solvent Extraction in Columns," Sep. Sci. Technol., 25, 16891707 (1990).

Leonard, R. A., D. G. Wygmans, M. J. McElwee, M. O. Wasserman, and G. F. Vandegrift, "The Centrifugal Contactor as a Concentrator in Solvent Extraction Processes," paper presented at the Seventh Symposium on Separation Science and Technology for Energy Applications, Knoxville, Tennessee, October 20-24,1991, submitted for publication in Sep. Sci. Technol. (1992).

Schulz, W. W. and E. P. Horwitz, "The TRUEX Process and the Management of Liquid TRU Waste," Sep. Sci. Technol., 23, 1191-1210 (1988).

Siddall, T. H., "Solvent Extraction Processes Based on Tri-n-butyl Phosphate," Chapter V in Chemical Processing of Reactor Fuels, J. F. Flagg, editor, Academic Press, New York, 199-248 (1961).

Treybal, R. E., Liquid Extraction, 2nd ed., McGraw-Hill Book Company, Inc., New York (1963).

Vandegrift, G. F., R. A. Leonard, M. J. Steindler, E. P. Horwitz, L. J. Basile, H. Diamond, D. G. Kalina, and L. Kaplan, "Transuranic Decomtamination of Nitric Acid Solutions by the TRUEX Solvent Extraction Process-Preliminary Development Studies," Argonne National Laboratory, ANL-8445 (1984).

Walas, S. M., Chemical Process Equipment, Butterworths, Boston (1988). 


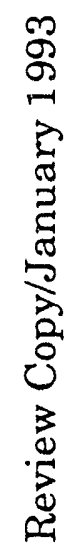

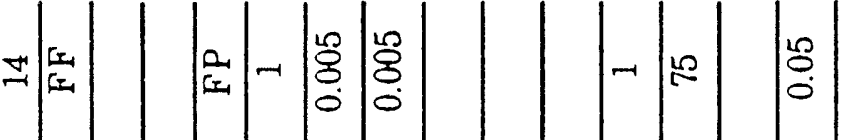

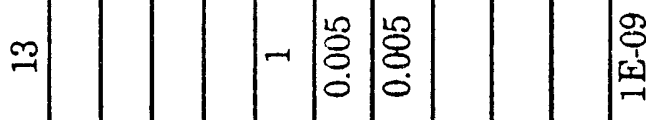

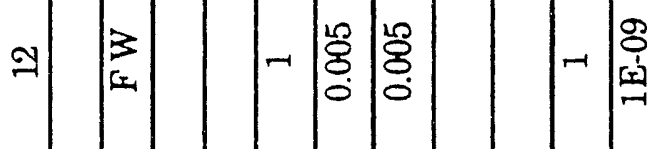

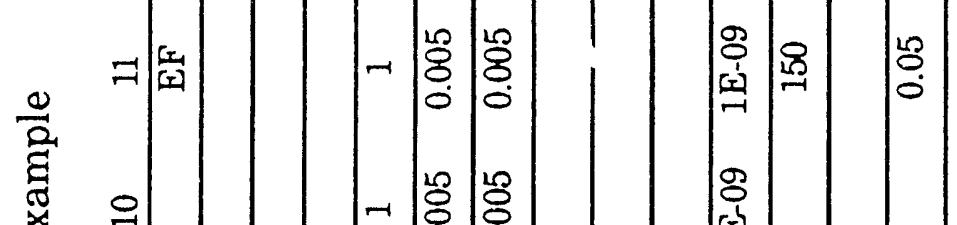

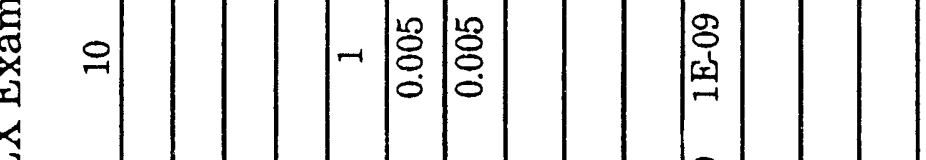

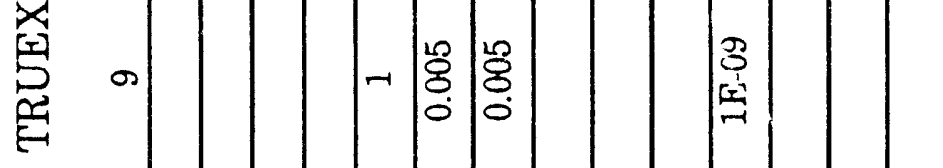

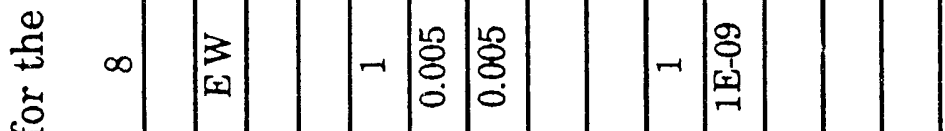

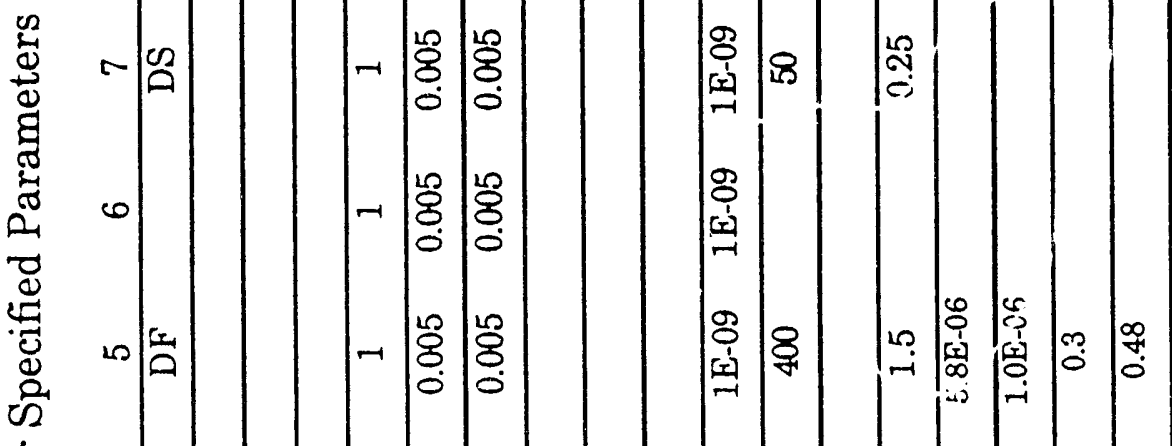

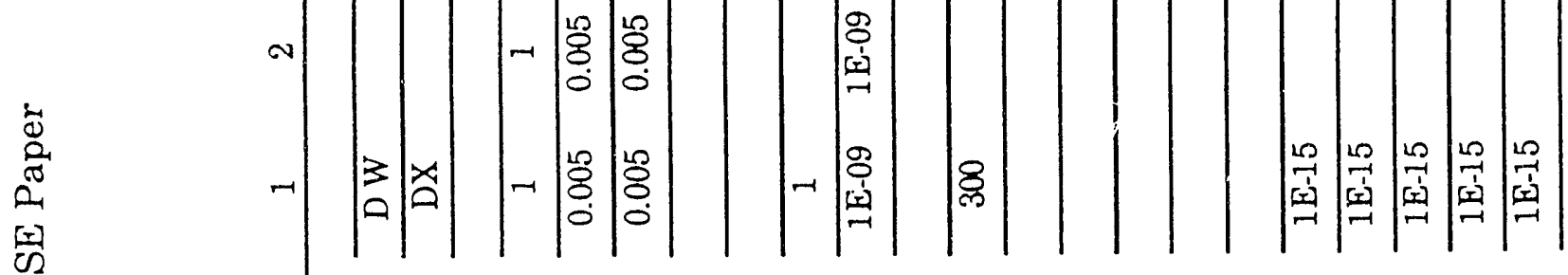

象

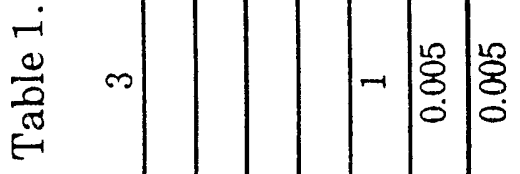

o

- 
2

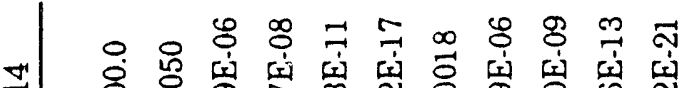

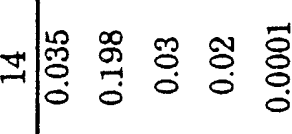

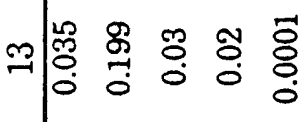

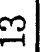

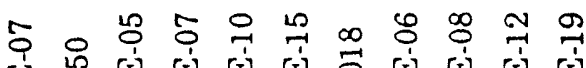
岳 员

ง|l

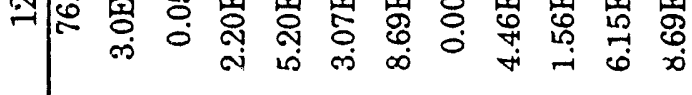

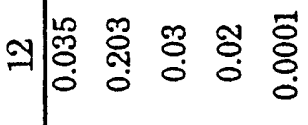

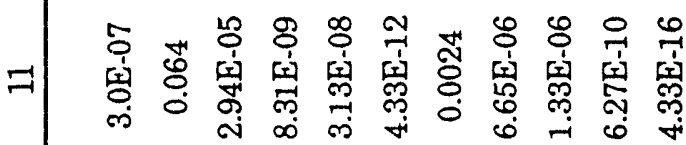

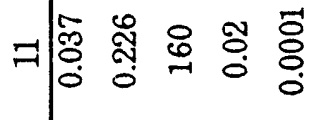

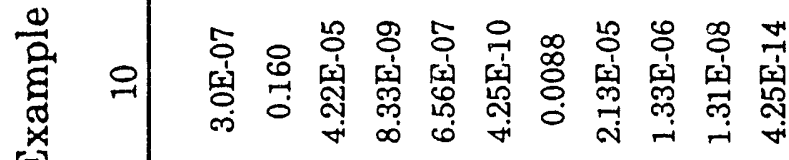

竞

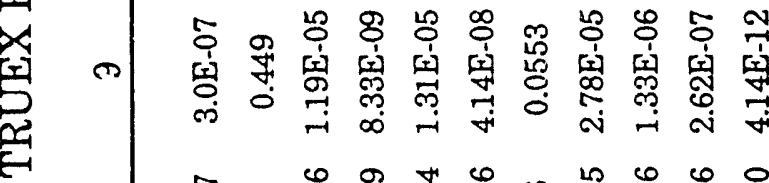

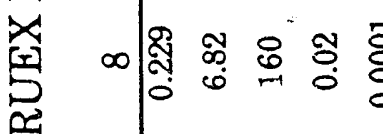

象

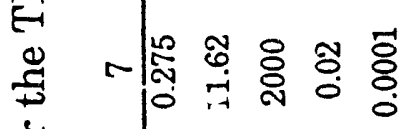

to

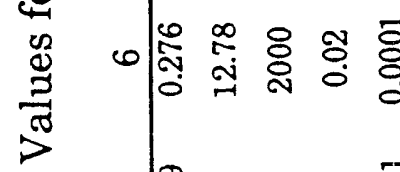

ص

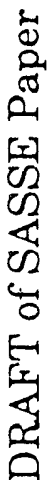

N|

\& $\infty \quad \infty$ 章

ڤ I

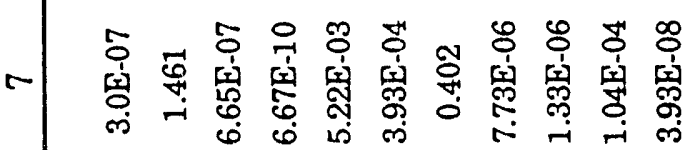

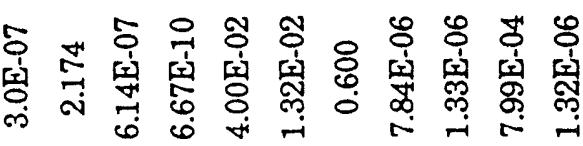

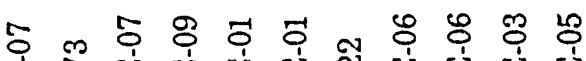

元

5 ○

炼

5 $ᄋ$ 우 궁

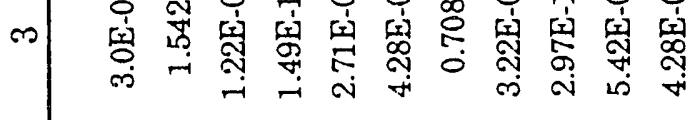

5

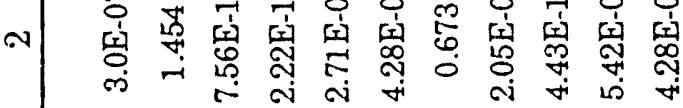

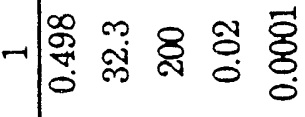

น

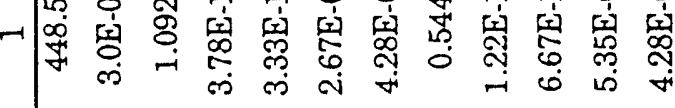

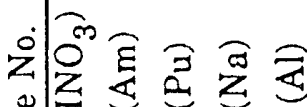

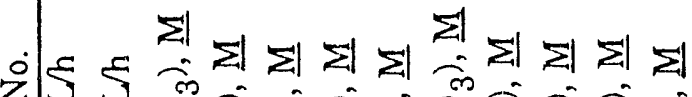

$\tilde{a}$

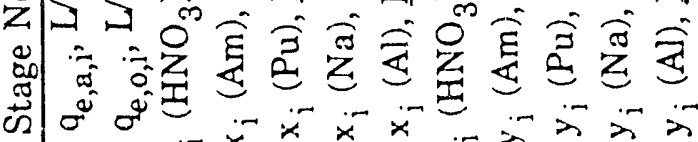




\section{Figure Captions}

Fig. 1. Schematic for Simple Stage Operation

Fig. 2. Schematic for Complex Stage Operation

Fig. 3. General Layout of a SASSE Worksheet

Fig. 4. TRUEX Process with 14 Stages 

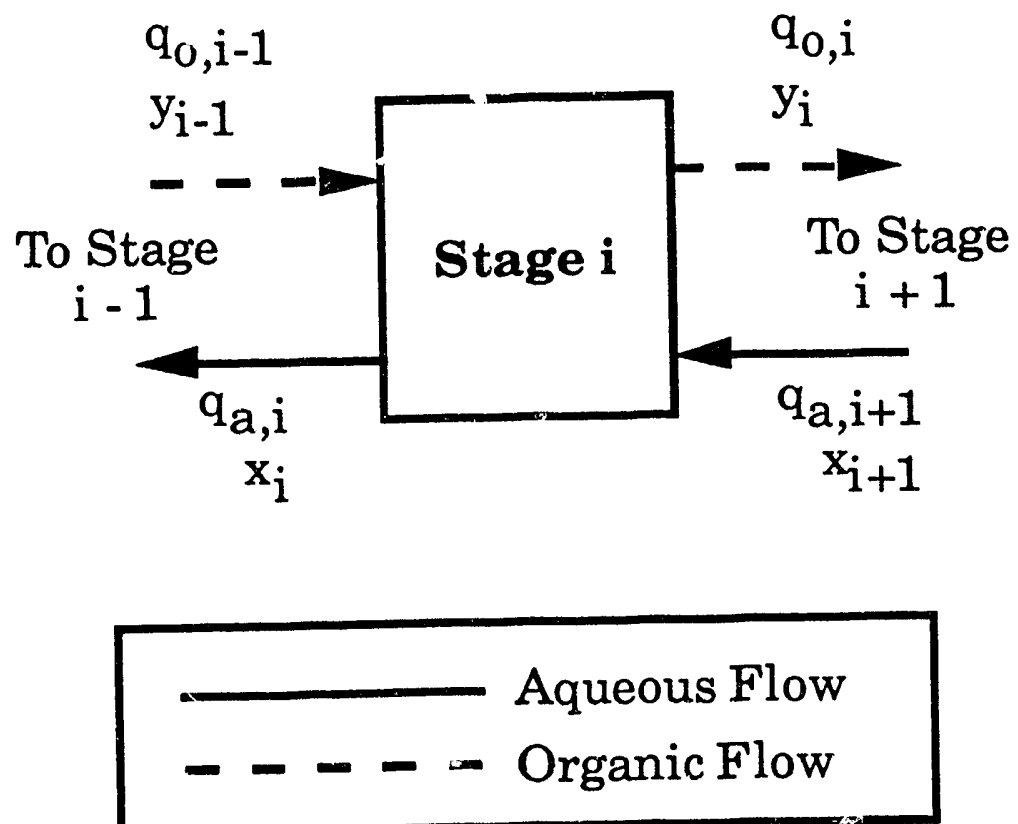

Fig. 1. Schematic for Simple Stage Operation 


$$
\begin{aligned}
& \mathrm{y}_{\mathrm{i}-1} \\
& \left(1-f_{e, o, i-1}\right) q_{o, i-1} \\
& \left(1-f_{e, o, i-1}\right) R_{o, i-1} q_{o, i-1}
\end{aligned}
$$

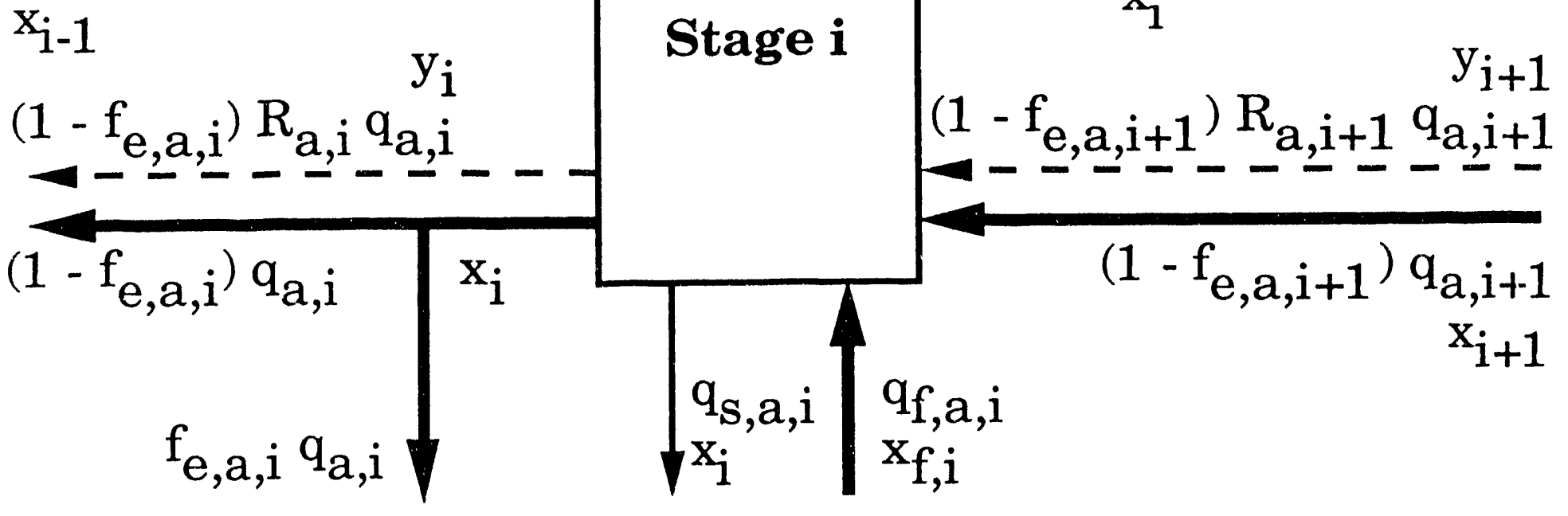

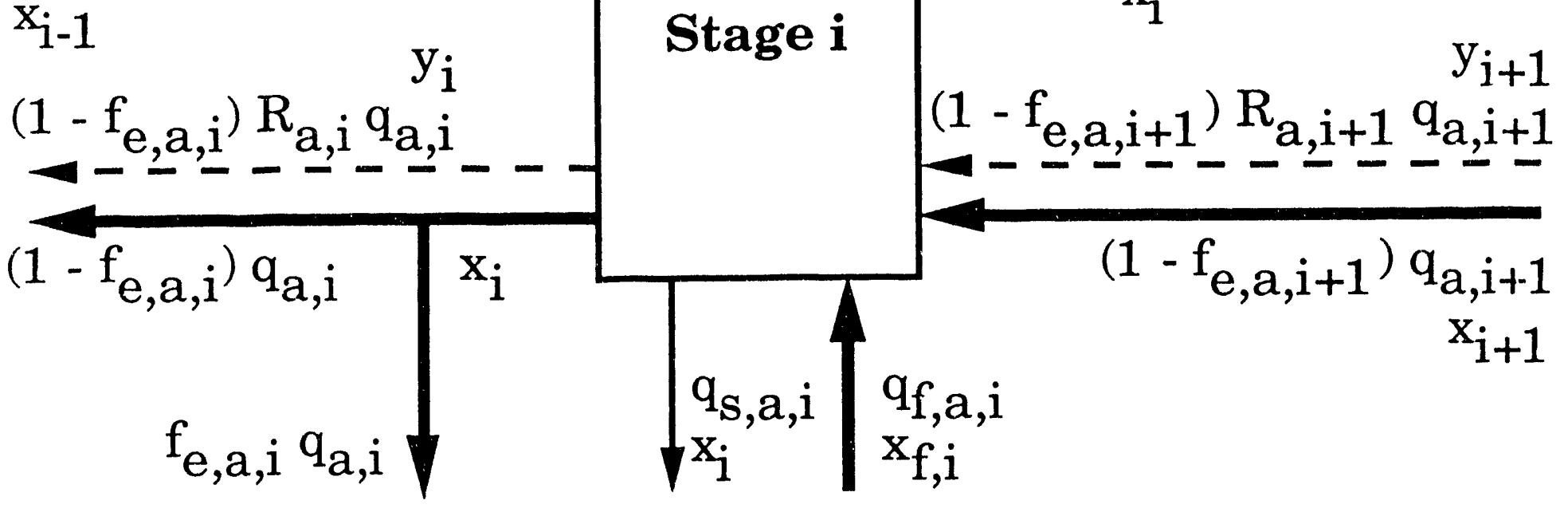

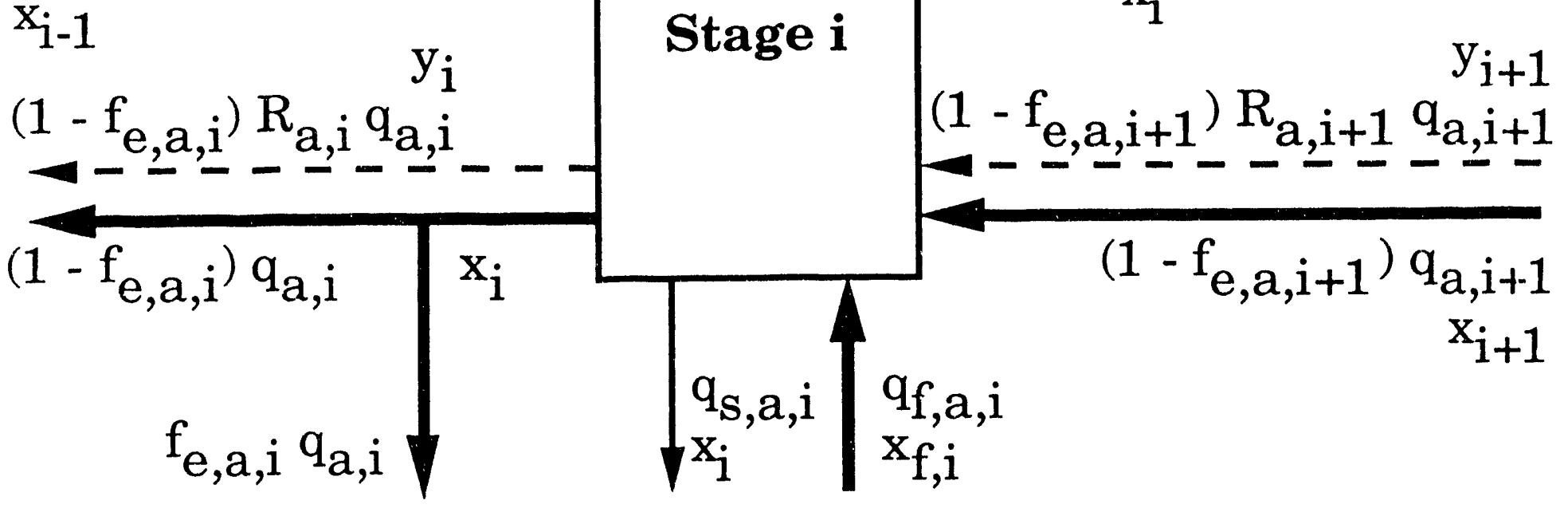

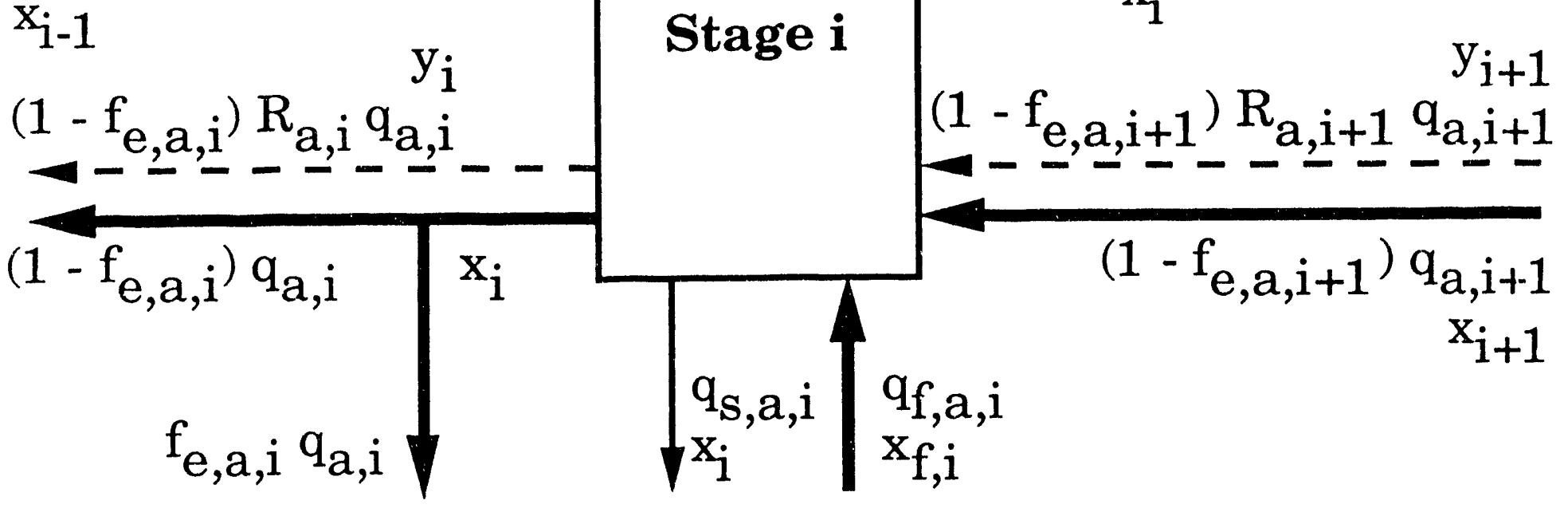

$$
\begin{aligned}
& \begin{array}{l}
q_{f, o, i} \uparrow q_{s, o, i} \\
y_{f, i}, f_{i}
\end{array}
\end{aligned}
$$

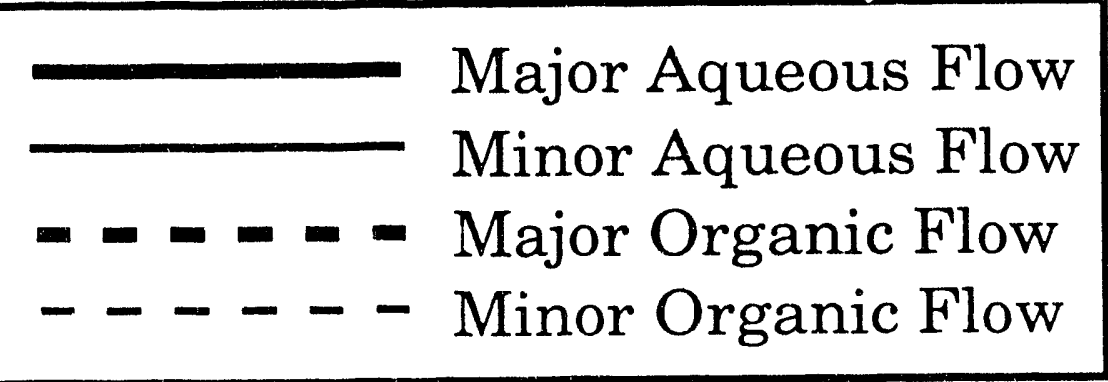

Fig. 2. Schematic for Complex Stage Operation 


\section{General Notes}

User Specified Parameters
D Values

Summary of the Calculations

Flow Balance Calculations

Mass Balance Calculations

Fig. 3. General Layout of a SASSE Worksheet 


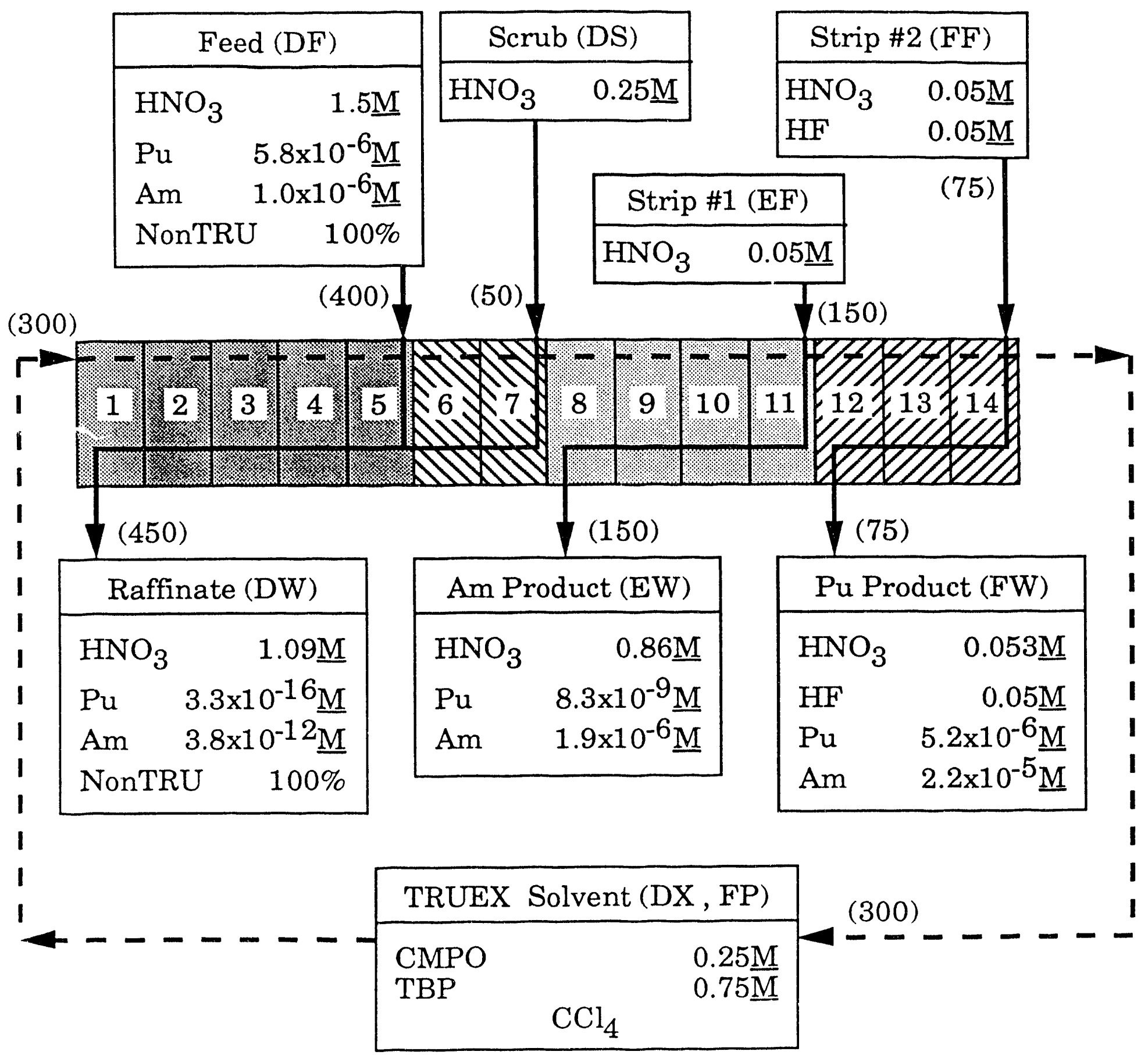

Fig. 4. TRUEX Process with 14 Stages 

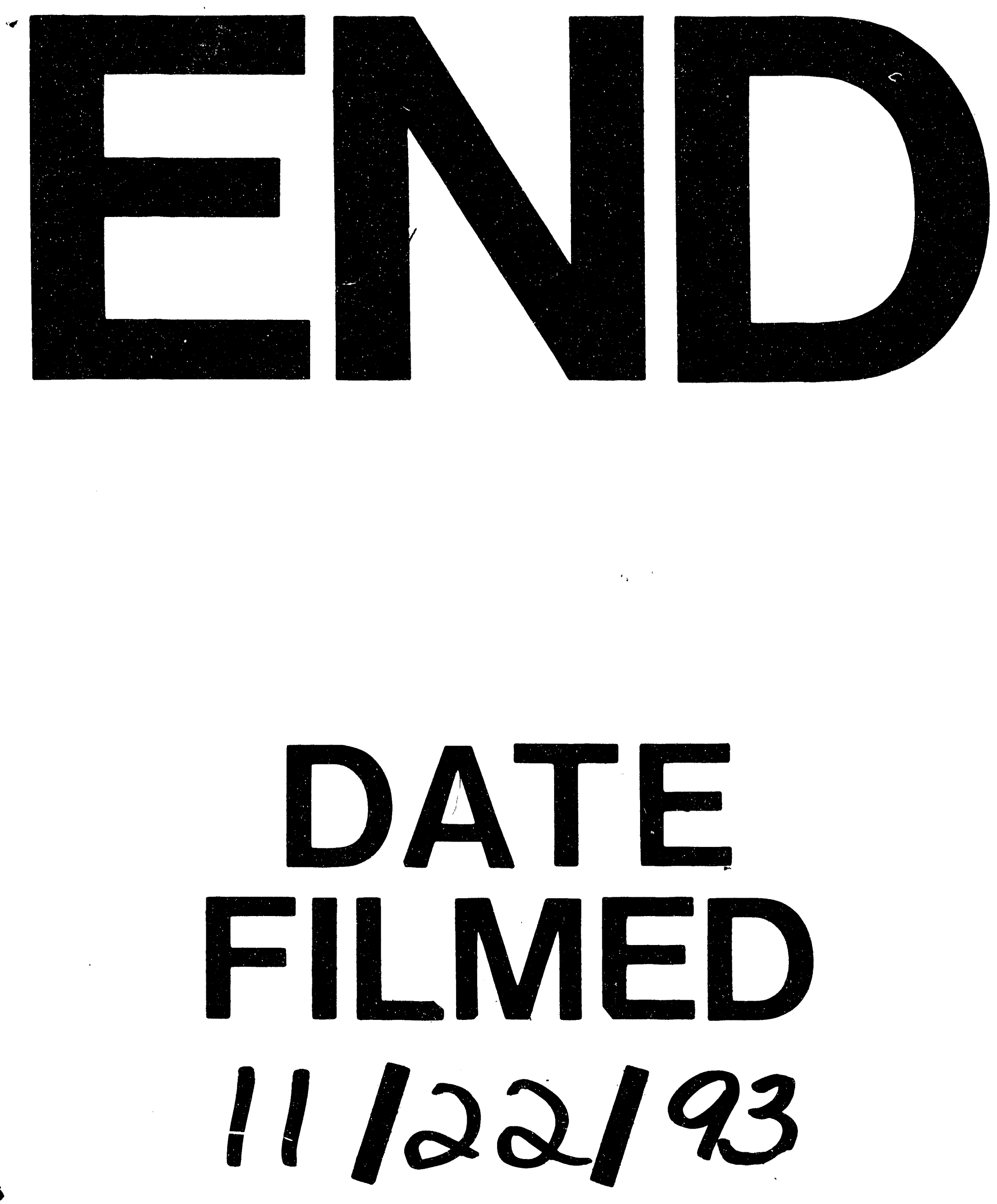

1 
